A-083

J. Chem. Thermodynamics 1979, 11, 247-260

\title{
Heat capacities of bis-tetraethylammonium tetrachloronickelate and tetrachlorozincate I. Structural transitions and thermophysical results ${ }^{a}$
}

\author{
CHRISTOPHER P. LANDEE ${ }^{b}$ and EDGAR F. WESTRUM, JR. \\ Department of Chemistry, The University of Michigan, Ann Arbor, \\ Michigan 48109, U.S.A.
}

(Received 13 February 1978; in revised form 27 June 1978)

\begin{abstract}
The heat capacities of bis-tetraethylammonium tetrachloronickelate (BEAN) and tetrachlorozincate (BEAZ) were measured over the temperature range 5 to $350 \mathrm{~K}$ by adiabatic calorimetry. Bifurcated anomalies were found for both compounds with the temperatures of the maxima occurring at $(222.1 \pm 0.1) \mathrm{K}$ and $(222.7 \pm 0.1) \mathrm{K}$ for BEAN and at $(227.9 \pm 0.2) \mathrm{K}$ and $(228.9 \pm 0.2) \mathrm{K}$ for BEAz. The excess entropies associated with the transition regions were 9.16 and $9.96 \mathrm{cal}_{\mathrm{th}} \mathrm{K}^{-1} \mathrm{~mol}^{-1}$ respectively. Both transition regions were characterized by equilibrium times of several days. Selected thermal functions $C_{p}^{\circ}$, $S^{\circ}$, and $-\left\{G^{\circ}(T)-H^{\circ}(0)\right\} / T$ at $298.15 \mathrm{~K}$ are, respectively, $151.7,185.33$, and $92.16 \mathrm{cal}_{\text {th }} \mathrm{K}^{-1}$ $\mathrm{mol}^{-1}$ for BEAN and 153.0, 181.52, and $88.53 \mathrm{cal}_{\mathrm{th}} \mathrm{K}^{-1} \mathrm{~mol}^{-1}$ for BEAZ.
\end{abstract}

\section{Introduction}

In $1965 \mathrm{Ham}^{(1)}$ proposed a model of the dynamic Jahn-Teller effect in which electronlattice coupling, though insufficiently strong to produce a static distortion, could partially quench orbital angular momentum. $\mathrm{A} \mathrm{Ni}^{2+}$ ion in tetrahedral symmetry was suggested as one likely to exhibit such an effect.

A nearly tetrahedral $\mathrm{NiCl}_{4}^{2-}$ group was found in bis-tetraethylammonium tetrachloronickelate (BEAN) during the $300 \mathrm{~K}$ structure determination of that compound by Stucky, Folkers, and Kistenmacher. ${ }^{(2)}$ The powder susceptibility and singlecrystal anisotropies of this compound were measured between 90 and $300 \mathrm{~K}$ by Gerloch and Slade ${ }^{(3)}$ and revealed an anomaly in the crystal anisotropy at about $220 \mathrm{~K}$. This decrease in anisotropy was interpreted as indicating a change in the geometry of the complex ion. Gerloch and Slade's analysis of the susceptibilities predicted almost perfect tetrahedral coordination of the nickel ion below $220 \mathrm{~K}$. Quenching of the orbital angular momentum in $\mathrm{Ni}^{2+}$ would reduce splitting of the ${ }^{3} \mathrm{~T}_{1 \mathrm{~g}}$ ground state by the spin-orbit interaction. Should the quenching be sufficiently

${ }^{a}$ This research was supported in part by the Chemical Thermodynamics Program, Chemistry Division, National Science Foundation under Contract No. CHE-77-10049.

b Present address: Program of Chemical Physics, Washington State University, Pullman, Washington 99164, U.S.A. 
large, the excited states would become thermally accessible at temperatures low enough to produce recognizable contributions to the total heat capacity.

Heat-capacity measurements have been made on the tetrahedral nickel compound discussed above as well as on the isostructural ${ }^{(4,5)}$ zinc analog (BEAZ). The heat capacity of the latter provides an estimate of the non-electronic contributions to the heat capacity of the nickel compound. The discussion of these measurements in terms of Ham's theory will be published subsequently. ${ }^{(6)}$ This paper presents the experimental results and discusses the significance of the bifurcated transitions found near $220 \mathrm{~K}$ in both compounds.

\section{Experimental}

\section{SAMPLE PREPARATION}

Both the BEAN sample and the BEAZ sample were prepared by the technique of Gill and Nyholm. ${ }^{(7)}$ The BEAN sample consisted of approximately equal portions of powder and small flat dark-blue plates 1 to $5 \mathrm{~mm}$ in length. The BEAZ sample, white in color, was similarly composed. Chemical analysis for the metal and chlorine agreed to within 0.1 per cent for both compounds. Both compounds were crystallized from anhydrous ethanol, dried in a high vacuum, and handled in the anhydrous atmosphere of a glove box. Duplicate chemical analyses for all non-metallic constituents yielded mean mass percentages of the constituents of BEAN: $\mathrm{C},(41.75 \pm 0.05)$, (theor. 41.71); $\mathrm{H},(9.16 \pm 0.09$ ), (theor. 8.68); N, (6.12 \pm 0.08 ), (theor. 6.08); Cl, (30.45 \pm 0.06 ), (theor. 30.78). Those of BEAZ were: $\mathrm{C},(41.19 \pm 0.05$ ), (theor. 41.12); H, (8.72 \pm 0.09 ), (theor. 8.56); $\mathrm{N},(5.93 \pm 0.08)$, (theor. 6.00); Cl, (30.14 \pm 0.06 ), (theor. 30.34). The figures are estimates of the accuracy of the determinations by the commercial analysts employed. The magnitude of the average deviation from theoretical values of the analyses is thus 0.22 per cent for BEAN and only 0.12 per cent for BEAZ and, therefore, slightly exceeds the uncertainties in the individual determinations themselves. Both samples are clearly of the claimed stoichiometry and the high hydrogen determinations are probably evidence that insufficient precautions were taken by the analysts in protecting the hygroscopic nickel compound from water absorption.

\section{HEAT-CAPACITY MEASUREMENTS}

Heat-capacity measurements were made in the Mark II adiabatic cryostat described elsewhere. ${ }^{(8)}$ The calorimeter, laboratory designation W-52, was a gold-plated OFHC copper can of internal volume $59.11 \mathrm{~cm}^{3}$. Table 1 lists the mass of sample used, pressure of helium exchange gas in the calorimeter, and molar masses used to convert

TABLE 1. Sample loading data; $m$ is the sample mass, $M$ is the molar mass of the sample, and $p$ the partial pressure of the helium used in the calorimeter

\begin{tabular}{lcccc}
\hline \multicolumn{1}{c}{ Compound } & Formula & $m / \mathrm{g}$ & $M / \mathrm{g}$ & $p / \mathrm{kPa}$ \\
\hline BEAN & $\mathrm{R}_{2} \mathrm{NiCl}_{4}$ & 36.1019 & 461.03 & 11.1 \\
BEAZ & $\mathrm{R}_{2} \mathrm{ZnCl}_{4}$ & 22.6082 & 467.70 & 6.9 \\
\hline
\end{tabular}


the experimental results to molar quantities. A density of $1.25 \mathrm{~g} \mathrm{~cm}^{-3}$ was used for the buoyancy correction. ${ }^{(2)}$ The results are presented in terms of the IPTS-48 temperature scale. ${ }^{(9)}$

During measurements in the regions of the transition temperature, special care was taken to adjust the adiabatic shields for minimal net thermal exchange between the shields and calorimeter. This action was necessitated by the long equilibrium times in this region.

\section{Results and discussion}

\section{THERMOPHYSICAL FUNCTIONS}

The experimental heat capacities for BEAN and BEAZ are shown in figure 1. The $C_{p}$ values have a normal sigmate shape except for the large sharp bifurcated anomalies occurring at $(222.1 \pm 0.1)$ and $(222.7 \pm 0.1) \mathrm{K}$ for BEAN and $(227.9 \pm 0.1)$ and $(228.9 \pm 0.2) \mathrm{K}$ for BEAZ. In figure 2 these anomalies are shown in more detail.

The heat capacities of BEAN and BEAZ are given also in table 2 in chronological sequence so that the temperature increments can be deduced approximately from the adjacent mean temperatures. Series of points have had slight adjustments made for curvature. The direct enthalpy determinations through the transition regions have been summarized in table 3 .

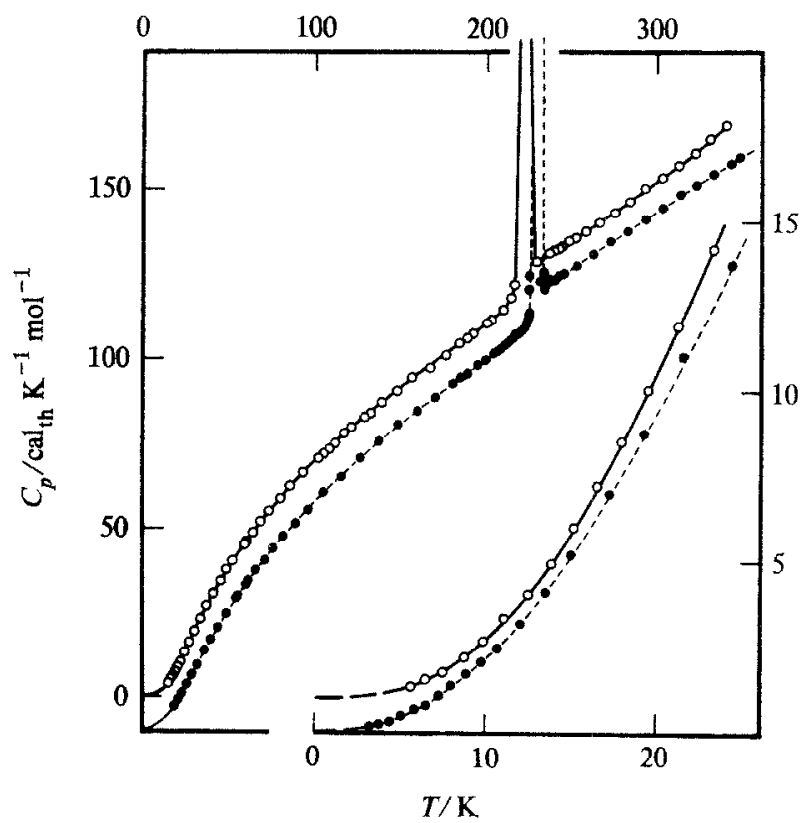

FIGURE 1. The experimental heat capacities: 0 , BEAN; - , BEAZ. For clarity, points in the transition region have been omitted (but see figure 2). The BEAZ curves have been displaced downward by $10 \mathrm{cal}_{\mathrm{th}} \mathrm{K}^{-1} \mathrm{~mol}^{-1}$ against the scale at the left and $1 \mathrm{cal}_{\mathrm{th}} \mathrm{K}^{-1} \mathrm{~mol}^{-1}$ against the scale at the right to separate the curves. 
TABLE 2. Experimental molar heat capacities of $\left[\left(\mathrm{CH}_{3} \mathrm{CH}_{2}\right)_{4} \mathrm{~N}\right]_{8} \mathrm{MCl}_{4}$ compounds $\left(\mathrm{cal}_{\mathrm{th}}=4.184 \mathrm{~J}\right)$

$\frac{\langle T\rangle}{\mathrm{K}} \frac{C_{p}}{\mathrm{cal}_{\mathrm{th}} \mathrm{K}^{-1} \mathrm{~mol}^{-1}} \quad \frac{\leq T\rangle}{\mathrm{K}} \frac{C_{p}}{\mathrm{cal}_{\mathrm{th}} \mathrm{K}^{-1} \mathrm{~mol}^{-1}} \quad \frac{\langle T\rangle}{\mathrm{K}} \frac{C_{p}}{\mathrm{cal}_{\mathrm{th}} \mathrm{K}^{-1} \mathrm{~mol}^{-1}} \quad \frac{<T>}{\mathrm{K}} \frac{C_{p}}{\mathrm{cal}_{\mathrm{th}} \mathrm{K}^{-1} \mathrm{~mol}^{-1}}$

\begin{tabular}{|c|c|c|c|c|c|c|c|}
\hline \multicolumn{8}{|c|}{ bis-Tetraethylammonium tetrachloronickelate (BEAN) } \\
\hline \multicolumn{2}{|c|}{ Series I } & \multirow{2}{*}{$\begin{array}{l}220.01 \\
220.64\end{array}$} & \multirow{2}{*}{$\begin{array}{l}285.8 \\
333.7\end{array}$} & \multirow{2}{*}{$\begin{array}{l}221.19 \\
221.45\end{array}$} & \multirow{2}{*}{$\begin{array}{l}542 \\
813\end{array}$} & \multirow{2}{*}{$\begin{array}{l}217.25 \\
218.35\end{array}$} & \multirow{2}{*}{$\begin{array}{l}120.02 \\
142.7\end{array}$} \\
\hline 104.80 & 71.96 & & & & & & \\
\hline 108.30 & 73.13 & 221.00 & 1668 & 221.56 & 243 & 219.31 & 170.0 \\
\hline 117.71 & 78.15 & 221.24 & 569 & 221.97 & 998 & 220.15 & 201.9 \\
\hline 129.02 & 83.08 & 221.62 & 512 & 222.11 & 1052 & 220.73 & 461 \\
\hline 138.95 & 87.16 & 222.07 & 406 & 222.30 & 659 & 221.10 & 510 \\
\hline 148.48 & 90.89 & 222.45 & 757 & 222.49 & 1275 & 221.51 & 278 \\
\hline 157.83 & 94.56 & 222.74 & 741 & 222.65 & 966 & 221.86 & 1863 \\
\hline 167.05 & 97.82 & 223.04 & 634 & 222.93 & 504 & 221.99 & 1184 \\
\hline 176.05 & 101.04 & 223.42 & 477 & 223.37 & 347 & 222.20 & 734 \\
\hline 184.92 & 104.39 & 223.95 & 315.5 & 223.92 & 294.5 & 222.42 & 918 \\
\hline 193.56 & 107.68 & 224.74 & 182.6 & 224.75 & 144.11 & 222.64 & 807 \\
\hline 202.00 & 110.92 & 225.85 & 135.4 & 225.66 & 127.35 & 222.76 & 7822 \\
\hline 210.25 & 114.54 & 229.24 & 128.51 & 226.42 & 127.56 & 222.95 & 511 \\
\hline 216.60 & 241.4 & 234.77 & 130.18 & 227.00 & 127.44 & 223.37 & 345 \\
\hline 220.05 & 504 & & & & & 223.93 & 232 \\
\hline \multicolumn{2}{|c|}{$\Delta H_{\mathrm{t}}$ Detn. $\mathrm{A}^{a}$} & \multicolumn{2}{|c|}{ Series V } & \multicolumn{2}{|c|}{ Series IX } & 224.73 & 167.6 \\
\hline 239.63 & 131.65 & 59.04 & 45.88 & 5.67 & 0.34 & 225.73 & 138.7 \\
\hline 248.94 & 134.66 & 63.60 & 49.02 & 6.59 & 0.52 & 226.84 & 125.21 \\
\hline 258.06 & 137.40 & 68.42 & 52.21 & 7.51 & 0.75 & 227.98 & 127.79 \\
\hline 266.97 & 140.51 & 73.52 & 55.21 & 8.74 & 1.22 & & \\
\hline 275.74 & 143.33 & 79.30 & 58.78 & 9.82 & 1.75 & \multicolumn{2}{|c|}{ Series $\mathrm{XII}^{\alpha}$} \\
\hline 284.37 & 146.46 & 85.71 & 62.53 & 11.06 & 2.38 & 204.86 & 111.91 \\
\hline 293.58 & 150.02 & 93.22 & 66.45 & 12.46 & 3.07 & 206.11 & 112.57 \\
\hline 303.38 & 153.5 & 102.20 & 70.85 & 13.79 & 3.98 & \multicolumn{2}{|c|}{$\Delta H_{\mathrm{t}}$ Detn. I } \\
\hline 312.98 & 157.4 & 111.29 & 75.14 & 15.04 & 5.06 & \multicolumn{2}{|c|}{$\Delta H_{\mathrm{t}}$ Detn. $\mathrm{J}$} \\
\hline 322.42 & 161.1 & 121.11 & 79.68 & 16.46 & 6.21 & \multirow{2}{*}{\multicolumn{2}{|c|}{ Series XIII ${ }^{a}$}} \\
\hline 331.69 & 164.4 & 131.44 & 84.10 & 17.92 & 7.59 & & \\
\hline \multirow[t]{3}{*}{340.80} & 168.9 & \multirow{2}{*}{\multicolumn{2}{|c|}{ Series VI }} & 19.44 & 9.10 & 211.90 & 115.99 \\
\hline & \multirow[b]{2}{*}{ Series II } & & & 21.24 & 10.98 & 218.79 & 219.4 \\
\hline & & \multicolumn{2}{|c|}{$\Delta H_{\mathrm{t}}$ Detn. $\mathrm{C}$} & 23.34 & 13.21 & \multirow{2}{*}{\multicolumn{2}{|c|}{$\begin{array}{ll}221.62 & 1019 \\
\Delta H_{\mathrm{t}} \text { Detn. } \mathrm{K}\end{array}$}} \\
\hline 189.28 & 105.99 & & & 25.92 & 16.07 & & \\
\hline 200.86 & 110.56 & & es VII & 29.14 & 19.69 & & \\
\hline$\Delta H_{\mathrm{t}}$ Det & & 189.61 & 106.03 & 32.67 & 23.33 & & $\mathbf{S} \mathbf{X T V}^{a}$ \\
\hline & & 200.28 & 110.26 & 36.33 & 27.04 & $\Delta H_{\mathrm{t}} \mathrm{De}$ & \\
\hline & es III & $\Delta H_{\mathrm{t}} \mathrm{De}$ & & 40.20 & 30.68 & & \\
\hline 202.89 & 111.30 & & & 44.22 & 34.32 & & es $X^{a}$ \\
\hline 211.01 & 114.89 & & es VIII & 48.51 & 37.92 & $\Delta H_{\mathrm{t}} \mathrm{De}$ & \\
\hline 217.29 & 239.6 & 204.38 & 111.93 & 53.32 & 41.66 & & \\
\hline 220.91 & 428 & 210.26 & 114.42 & 58.62 & 45.53 & & $\mathrm{XVI}{ }^{a}$ \\
\hline 224.56 & 235.5 & 213.31 & 115.61 & & & $\Delta H_{\mathrm{t}} \mathrm{De}$ & \\
\hline 230.52 & 128.9 & 214.44 & 116.50 & & ries $\mathrm{X}$ & & \\
\hline 237.82 & 131.06 & 215.56 & 117.33 & $\Delta H_{\mathrm{t}} \mathrm{De}$ & & & s XVII ${ }^{\alpha}$ \\
\hline & & 216.67 & 118.21 & $\Delta H_{\mathrm{t}} \mathrm{De}$ & & 211.34 & 115.10 \\
\hline & es IV & 217.75 & 126.63 & $\Delta H_{\mathrm{t}} \mathrm{De}$ & & 218.27 & 239.0 \\
\hline 208.53 & 113.78 & 218.70 & 165.9 & $\Delta H_{\mathrm{t}} \mathrm{De}$ & & & \\
\hline 214.35 & 118.97 & 219.45 & 231.0 & & & & XVIII ${ }^{a}$ \\
\hline 217.78 & 153.13 & 220.04 & 305.2 & & ies XI & 212.04 & 115.43 \\
\hline 218.73 & 243.9 & 220.52 & 366.4 & 214.89 & 116.23 & $\Delta H_{\mathrm{t}} \mathrm{De}$ & \\
\hline 219.42 & 315.8 & 220.88 & 559 & 216.08 & 117.37 & $\Delta H_{\mathrm{t}} \mathrm{De}$ & \\
\hline
\end{tabular}


TABLE 2-continued

\begin{tabular}{|c|c|c|c|c|c|c|c|}
\hline$\frac{<T>}{\mathrm{K}}$ & $\frac{C_{p}}{\mathrm{cal}_{\mathrm{th}} \mathrm{K}^{-1} \mathrm{~mol}^{-1}}$ & $\frac{\langle T\rangle}{\mathrm{K}}$ & $\frac{C_{p}}{\mathrm{~K}^{-1} \mathrm{~mol}^{-1}}$ & $\frac{<T>}{K}$ & $\frac{C_{p}}{\mathrm{cal}_{\mathrm{th}} \mathrm{K}^{-1} \mathrm{~mol}^{-1}}$ & $\frac{<T>}{\mathbf{K}}$ & $\frac{C_{p}}{\mathrm{cal}_{\mathrm{th}} \mathrm{K}^{-1} \mathrm{~mol}^{-1}}$ \\
\hline \multicolumn{8}{|c|}{ bis-Tetraethylammonium tetrachlorozincate (BEAz) } \\
\hline & Series I & 228.46 & 1226 & 5.44 & 0.291 & 205.64 & 111.86 \\
\hline 241.99 & 134.17 & 229.65 & 444 & 6.17 & 0.430 & 212.87 & 114.76 \\
\hline 252.66 & 137.58 & 232.23 & 179.1 & 6.93 & 0.631 & 217.83 & 117.04 \\
\hline 262.74 & 140.93 & 236.06 & 132.38 & 7.60 & 0.761 & 220.91 & 118.01 \\
\hline 272.92 & 144.25 & 240.27 & 133.51 & 8.30 & 1.023 & 221.87 & 118.62 \\
\hline 283.25 & 147.83 & \multirow{2}{*}{\multicolumn{2}{|c|}{ Series III }} & 9.11 & 1.319 & 222.82 & 119.10 \\
\hline 293.47 & 151.19 & & & 9.92 & 1.675 & 223.76 & 121.70 \\
\hline 303.50 & 154.79 & 200.45 & 109.38 & 10.73 & 2.104 & 224.69 & 120.66 \\
\hline 313.38 & 158.36 & $\Delta H_{\mathrm{t}} \mathrm{De}$ & A & 11.79 & 2.452 & 225.62 & 122.74 \\
\hline 323.28 & 161.52 & 245.38 & 135.19 & 13.05 & 3.171 & 226.52 & 134.60 \\
\hline 333.20 & 164.56 & & & 14.45 & 4.121 & 227.20 & 269.86 \\
\hline 342.98 & 167.77 & \multicolumn{2}{|c|}{ Series IV } & 16.00 & 5.217 & 227.51 & 731 \\
\hline 348.73 & 169.70 & 208.50 & 112.99 & 18.19 & 7.035 & 227.68 & 745 \\
\hline & Series II & 214.26 & 115.43 & 20.11 & 8.784 & 227.96 & 455 \\
\hline 53.70 & $\begin{array}{r}39.35 \\
\text { sentes II }\end{array}$ & 219.65 & 117.91 & 22.46 & 11.014 & 228.21 & 1123 \\
\hline 60.28 & 44.03 & 223.39 & 119.65 & 25.26 & 13.726 & 228.42 & 632 \\
\hline 65.53 & $\begin{array}{l}4.03 \\
47.63\end{array}$ & 225.51 & 123.75 & 27.99 & 16.52 & 228.64 & 943 \\
\hline 70.69 & 50.86 & 227.16 & 267.7 & 31.09 & 19.69 & 228.81 & 1100 \\
\hline 75.86 & $\begin{array}{l}50.00 \\
54.03\end{array}$ & 228.00 & 830 & 35.01 & 23.52 & 228.97 & 1041 \\
\hline 81.63 & 57.71 & 228.46 & 801 & 38.99 & 27.20 & 229.12 & 660 \\
\hline 88.14 & 61.64 & 228.93 & 818 & 43.25 & 30.92 & 229.26 & 1136 \\
\hline 95.77 & 65.61 & 229.38 & 853 & 47.98 & 34.80 & 229.42 & 1101 \\
\hline 105.28 & 70.52 & 229.84 & 761 & 53.48 & 39.05 & 229.57 & 1063 \\
\hline 115.56 & 75.55 & 230.44 & 518 & 59.84 & 43.74 & 229.74 & 962 \\
\hline 126.00 & 80.45 & 231.43 & 236.80 & & & 230.05 & 351.40 \\
\hline 137.43 & $\begin{array}{l}80.49 \\
85.45\end{array}$ & 233.05 & 135.73 & \multirow{2}{*}{\multicolumn{2}{|c|}{ Series VII }} & 230.31 & 776 \\
\hline 149.01 & $\begin{array}{l}90.20 \\
90.20\end{array}$ & 235.00 & 131.80 & & & 230.52 & 507 \\
\hline 159.76 & 94.40 & 236.96 & 132.44 & 104.26 & 69.91 & 230.81 & 216.8 \\
\hline 170.02 & 98.37 & & \multicolumn{2}{|c|}{$\begin{array}{l}104.26 \\
\Delta H_{\mathrm{t}} \text { Detn. } \mathrm{D}^{69.91}\end{array}$} & 231.41 & 249.8 \\
\hline 180.13 & 102.10 & \multirow{2}{*}{\multicolumn{2}{|c|}{$\begin{array}{cc} & \text { Series V } \\
199.75 & 109.49\end{array}$}} & \multicolumn{2}{|c|}{$\Delta H_{\mathrm{t}}$ Detn. $\mathrm{E}$} & 232.04 & 183.8 \\
\hline 189.29 & $\begin{array}{l}102.10 \\
105.56\end{array}$ & 199.75 & & 199.14 & 109.38 & 232.83 & 132.74 \\
\hline 199.39 & $\begin{array}{l}105.56 \\
10938\end{array}$ & \multicolumn{2}{|c|}{$\Delta H_{\mathrm{t}}$ Detn. B } & \multicolumn{2}{|c|}{$\Delta H_{\mathrm{t}}$ Detn. F } & 233.71 & 132.74 \\
\hline 206.73 & $\begin{array}{l}109.38 \\
112.03\end{array}$ & \multicolumn{2}{|c|}{$\Delta H$, Detn. C } & \multicolumn{2}{|c|}{$248.28 \quad 136.08$} & 234.59 & 130.97 \\
\hline 211.43 & $\begin{array}{l}112.03 \\
114.30\end{array}$ & 242.53 & 134.37 & & & 235.47 & 134.17 \\
\hline 216.06 & 116.36 & \multicolumn{2}{|c|}{ Series VI } & \multicolumn{2}{|r|}{ Series VIII } & 236.35 & 132.89 \\
\hline 225.05 & 130.39 & 4.28 & 0.139 & 185.68 & 104.18 & 237.22 & 133.53 \\
\hline 227.65 & 879 & 4.81 & 0.198 & 195.79 & 108.02 & & \\
\hline
\end{tabular}

${ }^{a}$ These determinations are considered to have been made on the sample incompletely converted to the low-temperature phase. Not included in final fit.

The experimental heat capacities in non-transition regions were curvature corrected and fitted to polynomials in reduced temperature by the method of least squares and integrated to yield values of the thermodynamic functions at selected temperature intervals. Within the transition regions, the thermal functions were obtained by numerical integration of heat-capacity points checked against large-scale plots. Values for the thermal functions thus obtained are presented in table 4 for both compounds. Entropy and enthalpy increments below the lowest measured temperature were obtained by a Debye limiting-law extrapolation. 


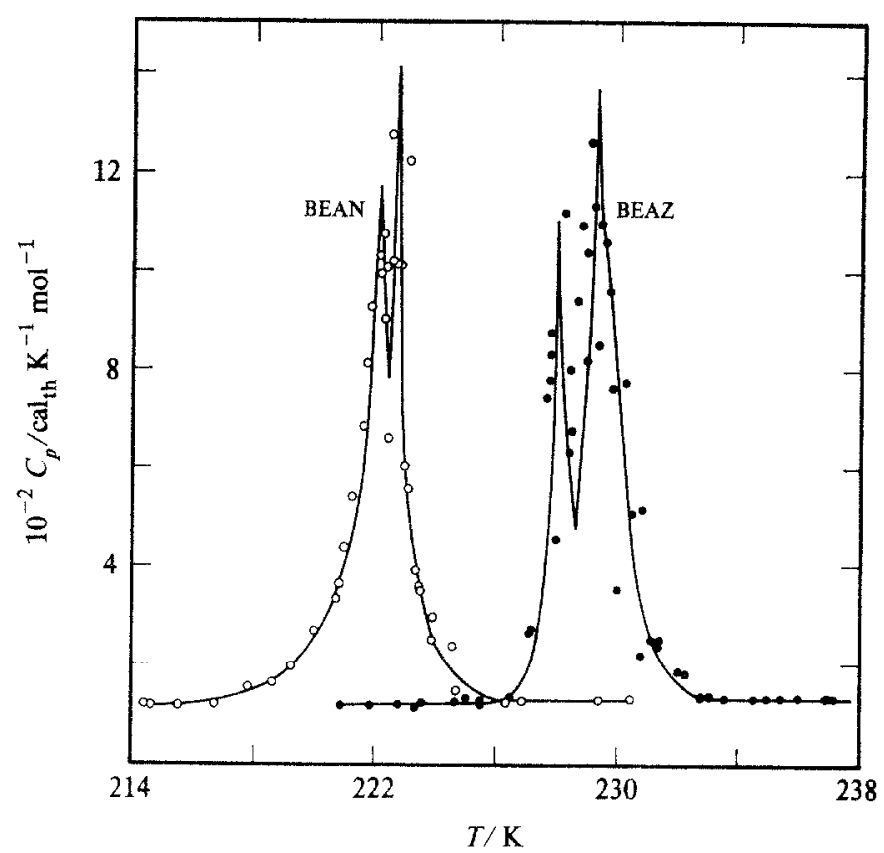

FIGURE 2. Experimental heat capacities in the transition regions. O, BEAN;, BEAZ. Many points have been omitted (compare footnote $a$, table 2).

TABLE 3. Enthalpy increments of $\left[\left(\mathrm{CH}_{3} \mathrm{CH}_{2}\right)_{4} \mathrm{~N}_{2} \mathrm{MCl}_{4}\right.$ compounds $\left(\mathrm{cal}_{\mathrm{th}}=4.184 \mathrm{~J}\right)$

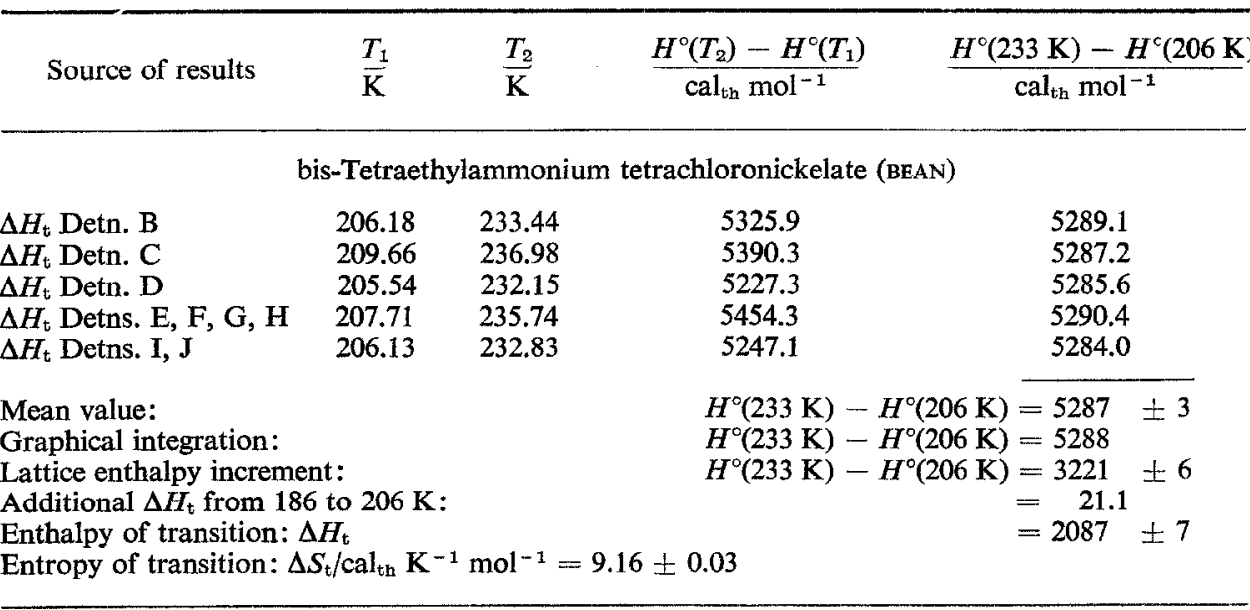


TABLE 3-continued

\begin{tabular}{|c|c|c|c|c|}
\hline Source of results & $\frac{T_{1}}{\hat{K}}$ & $\frac{T_{2}}{\mathrm{~K}}$ & $\frac{H^{\circ}\left(T_{2}\right)-H^{\circ}\left(T_{1}\right)}{\mathrm{cal}_{\mathrm{th}} \mathrm{mol}^{-1}}$ & $\frac{H^{\circ}(240 \mathrm{~K})-H^{\circ}(205 \mathrm{~K})}{\mathrm{cal}_{\text {th }} \mathrm{mol}^{-1}}$ \\
\hline \multicolumn{5}{|c|}{ bis-Tetraethylammonium tetrachlorozincate (BEAZ) } \\
\hline $\begin{array}{l}\text { Detn. A } \\
\text { Detns. B, C } \\
\text { Detn. D } \\
\text { Series II (11 detn.) } \\
\text { Series IV (16 detn.) } \\
\text { Series VII (37 detn.) }\end{array}$ & $\begin{array}{l}205.86 \\
203.54 \\
205.77 \\
204.37 \\
205.47 \\
200.78\end{array}$ & $\begin{array}{l}240.72 \\
241.59 \\
243.72 \\
242.37 \\
237.94 \\
237.66\end{array}$ & $\begin{array}{l}6449.7 \\
6829.8 \\
6857.4 \\
6670.9 \\
6119.1 \\
6608\end{array}$ & $\begin{array}{l}6449.2 \\
6454.7 \\
6444.9 \\
6450.3 \\
6446.6 \\
6450\end{array}$ \\
\hline \multicolumn{4}{|c|}{$\begin{array}{ll}\text { Mean value: } & H^{\circ}(240 \mathrm{~K}) \\
\text { Graphical integration: } & H^{\circ}(240 \mathrm{~K}) \\
\text { Lattice enthalpy increment: } & H^{\circ}(240 \mathrm{~K}) \\
\text { Enthalpy of transition: } \Delta H_{\mathrm{t}} & \\
\text { Entropy of transition: } \Delta S_{\mathrm{t}} / \mathrm{cal}_{\mathrm{th}} \mathrm{K}^{-1} \mathrm{~mol}^{-1}=9.66 \pm 0.04\end{array}$} & $\begin{aligned}205 K) & =6449 \pm 4 \\
205 K) & =6451 \\
205 K) & =4165 \pm 6 \\
& =2284 \pm 8\end{aligned}$ \\
\hline
\end{tabular}

The results in the transition regions are of lower precision than the others due to the long equilibration times (several days) in these temperature ranges (see below). Adjustments were necessary to compute heat capacities from experimental results when measurements of the drift of temperature against time were abandoned before the sample reached complete thermal equilibration. (These adjustments are discussed more fully in the appendix.) The accuracy of these points is, however, considered to be better than 5 per cent near the top of the heat-capacity maxima and increases

TABLE 4. Thermodynamic properties of $\left[\left(\mathrm{CH}_{3} \mathrm{CH}_{2}\right)_{4} \mathrm{~N}\right]_{2} \mathrm{MCl}_{4}$ compounds

$$
\left(\mathrm{cal}_{\mathrm{th}}=4.184 \mathrm{~J}\right)
$$

\begin{tabular}{|c|c|c|c|c|}
\hline$\frac{T}{\mathrm{~K}}$ & $\frac{C_{p}}{\mathrm{cal}_{\mathrm{th}} \mathrm{K}^{-1} \mathrm{~mol}^{-1}}$ & $\frac{S^{\circ}(T)-S^{\circ}(0)}{\mathrm{cal}_{\mathrm{th}} \mathrm{K}^{-1} \mathrm{~mol}^{-1}}$ & $\frac{H^{\circ}(T)-H^{\circ}(0)}{\mathrm{cal}_{\mathrm{ta}} \mathrm{mol}^{-1}}$ & $\frac{-\left\{G^{\circ}(T)-H^{\circ}(0)\right\} / T}{\mathrm{cal}_{\mathrm{th}} \mathrm{K}^{-1} \mathrm{~mol}^{-\mathrm{I}}}$ \\
\hline \multicolumn{5}{|c|}{ bis-Tetraethylammonium tetrachloronickelate (BEAN) } \\
\hline \multicolumn{5}{|c|}{ Crystal III } \\
\hline 5 & 0.229 & $(0.079)$ & $(0.295)$ & $(0.020)$ \\
\hline 10 & 1.751 & 0.609 & 4.547 & 0.155 \\
\hline 15 & 4.984 & 1.875 & 20.694 & 0.495 \\
\hline 20 & 9.646 & 3.920 & 56.79 & 1.080 \\
\hline 25 & 15.09 & 6.649 & 118.46 & 1.911 \\
\hline 30 & 20.54 & 9.888 & 207.71 & 2.965 \\
\hline 35 & 25.71 & 13.446 & 323.44 & 4.205 \\
\hline 40 & 30.51 & 17.20 & 464.14 & 5.593 \\
\hline 45 & 34.96 & 21.05 & 627.9 & 7.096 \\
\hline 50 & 39.10 & 24.95 & 813.2 & 8.686 \\
\hline 60 & 46.58 & 32.75 & 1242.4 & 12.048 \\
\hline 70 & 53.20 & 40.44 & 1741.9 & 15.56 \\
\hline 80 & 59.17 & 47.94 & 2304.2 & 19.14 \\
\hline 90 & 64.65 & 55.23 & 2923.6 & 22.75 \\
\hline 100 & 69.76 & 62.31 & 3596.0 & 26.35 \\
\hline
\end{tabular}


TABLE 4-continued

\begin{tabular}{|c|c|c|c|c|}
\hline$\frac{T}{\mathrm{~K}}$ & $\frac{C_{p}}{\mathrm{cal}_{\llcorner\mathrm{L}} \mathrm{K}^{-1} \mathrm{~mol}^{-1}}$ & $\frac{S^{\circ}(T)-S^{\circ}(0)}{\mathrm{cal}_{\mathrm{th}} \mathrm{K}^{-1} \mathrm{~mol}^{-1}}$ & $\frac{H^{\circ}(T)-H^{\circ}(0)}{\mathrm{cal}_{\mathrm{tn}} \mathrm{mol}^{-1}}$ & $\frac{-\left\{G^{\circ}(T)-H^{\circ}(0)\right\} / T}{\mathrm{cal}_{\mathrm{th}} \mathrm{K}^{-1} \mathrm{~mol}^{-1}}$ \\
\hline 110 & 74.58 & 69.19 & 4317.9 & 29.93 \\
\hline 120 & 79.15 & 75.87 & 5087 & 33.48 \\
\hline 130 & 83.48 & 82.38 & 5900 & 37.00 \\
\hline 140 & 87.61 & 88.72 & 6756 & 40.47 \\
\hline 150 & 91.52 & 94.90 & 7652 & 43.89 \\
\hline 160 & 95.26 & 100.93 & 8586 & 47.27 \\
\hline 170 & 98.89 & 106.81 & 9556 & 50.60 \\
\hline 180 & 102.52 & 112.57 & 10563 & 53.88 \\
\hline 190 & 106.27 & 118.21 & 11607 & 57.12 \\
\hline 200 & 110.16 & 123.76 & 12689 & 60.31 \\
\hline \multicolumn{5}{|c|}{$C_{p}$ Maximum } \\
\hline \multicolumn{5}{|c|}{ Crystal II } \\
\hline \multicolumn{5}{|c|}{$C_{p}$ Maximum } \\
\hline \multicolumn{5}{|c|}{ Crystal I } \\
\hline 240 & 131.80 & 154.74 & 19560 & 73.25 \\
\hline 250 & 134.91 & 160.19 & 20893 & 76.62 \\
\hline 260 & 138.11 & 165.54 & 22258 & 79.93 \\
\hline 270 & 141.45 & 170.82 & 23656 & 83.20 \\
\hline 280 & 144.94 & 176.02 & 25087 & 86.42 \\
\hline 290 & 148.57 & 181.17 & 26555 & 89.60 \\
\hline 300 & 152.3 & 186.27 & 28059 & 92.74 \\
\hline 310 & 156.1 & 191.33 & 29601 & 95.84 \\
\hline 320 & 160.1 & 196.35 & 31182 & 98.90 \\
\hline 330 & 164.1 & 201.33 & 32803 & 101.93 \\
\hline 340 & 168.3 & 206.29 & 34465 & 104.93 \\
\hline 273.15 & 142.53 & 172.46 & 24103 & 84.22 \\
\hline 298.15 & 151.6 & 185.33 & 27778 & 92.16 \\
\hline \multicolumn{5}{|c|}{ bis-Tetraethylammonium tetrachlorozincate (BEAZ) } \\
\hline \multicolumn{5}{|c|}{ Crystal III } \\
\hline 5 & 0.225 & 0.079 & 0.295 & 0.020 \\
\hline 10 & 1.663 & 0.593 & 4.413 & 0.152 \\
\hline 15 & 4.472 & 1.750 & 19.149 & 0.474 \\
\hline 20 & 8.683 & 3.586 & 51.57 & 1.008 \\
\hline 25 & 13.464 & 6.034 & 106.86 & 1.760 \\
\hline 30 & 18.59 & 8.941 & 186.97 & 2.709 \\
\hline 35 & 23.48 & 12.177 & 292.23 & 3.827 \\
\hline 40 & 28.10 & 15.62 & 421.28 & 5.084 \\
\hline 45 & 32.42 & 19.18 & 572.7 & 6.451 \\
\hline 50 & 36.45 & 22.80 & 745.0 & 7.905 \\
\hline 60 & 43.82 & 30.11 & 1147.0 & 10.997 \\
\hline 70 & 50.49 & 37.38 & 1619.0 & 14.248 \\
\hline 80 & 56.69 & 44.53 & 2155.3 & 17.59 \\
\hline 90 & 62.48 & 51.54 & 2751.4 & 20.97 \\
\hline 100 & 67.90 & 58.41 & 3403.6 & 24.37 \\
\hline
\end{tabular}


TABLE 4-continued

\begin{tabular}{|c|c|c|c|c|}
\hline$\frac{T}{\mathbf{K}}$ & $\frac{C_{p}}{\mathrm{cal}_{\mathrm{tn}} \mathrm{K}^{-1} \mathrm{~mol}^{-1}}$ & $\frac{S^{\circ}(T)-S^{\circ}(0)}{\mathrm{cal}_{\mathrm{th}} \mathrm{K}^{-1} \mathrm{~mol}^{-1}}$ & $\frac{H^{\circ}(T)-H^{\circ}(0)}{\mathrm{cal}_{\text {th }} \mathrm{mol}^{-1}}$ & $\frac{-\left\{G^{\circ}(T)-H^{\circ}(0)\right\} / T}{\mathrm{cal}_{\mathrm{tb}} \mathrm{K}^{-1} \mathrm{~mol}^{-1}}$ \\
\hline 110 & 72.95 & 65.12 & 4108.1 & 27.77 \\
\hline 120 & 77.69 & 71.67 & 4861.6 & 31.16 \\
\hline 130 & 82.17 & 78.07 & 5661 & 34.52 \\
\hline 140 & 86.46 & 84.32 & 6504 & 37.86 \\
\hline 150 & 90.58 & 90.42 & 7390 & 41.16 \\
\hline 160 & 94.55 & 96.40 & 8316 & 44.43 \\
\hline 170 & 98.37 & 102.25 & 9280 & 47.66 \\
\hline 180 & 102.08 & 107.97 & 10283 & 50.85 \\
\hline 190 & 105.77 & 113.59 & 11322 & 54.00 \\
\hline 200 & 109.58 & 119.11 & 12398 & 57.12 \\
\hline 210 & 113.67 & 124.56 & 13514 & 60.20 \\
\hline 220 & 118.10 & 129.95 & 14673 & 63.25 \\
\hline \multirow[t]{2}{*}{227.9} & \multicolumn{3}{|c|}{$C_{p}$ Maximum } & \\
\hline & \multicolumn{3}{|c|}{ Crystal II } & \\
\hline \multirow[t]{2}{*}{228.9} & \multicolumn{3}{|c|}{$C_{p}$ Maximum } & \\
\hline & \multicolumn{3}{|c|}{ Crystal III } & \\
\hline 240 & 133.51 & 150.56 & 19407 & 69.70 \\
\hline 250 & 136.78 & 156.08 & 20759 & 73.04 \\
\hline 260 & 139.99 & 161.50 & 22143 & 76.34 \\
\hline 270 & 143.25 & 166.85 & 23559 & 79.59 \\
\hline 280 & 146.64 & 172.12 & 25008 & 82.80 \\
\hline 290 & 150.1 & 177.32 & 26492 & 85.97 \\
\hline 300 & 153.6 & 182.47 & 28011 & 89.10 \\
\hline 310 & 157.1 & 187.57 & 29564 & 92.20 \\
\hline 320 & 160.4 & 192.61 & 31152 & 95.26 \\
\hline 330 & 163.6 & 197.59 & 32772 & 98.28 \\
\hline 340 & 166.8 & 202.53 & 34425 & 101.28 \\
\hline 350 & 170.0 & 207.41 & 36109 & 104.24 \\
\hline 273.15 & 144.31 & 168.52 & 24012 & 80.61 \\
\hline 298.15 & 153.0 & 181.52 & 27727 & 88.53 \\
\hline
\end{tabular}

quickly as the heat capacity decreases to that of the lattice. Outside the transition regions, the accuracy is estimated to be within \pm 0.1 per cent.

A further impression of the accuracy may be gained from figure 3 showing the deviations of the experimental points from the smoothed results. The Schottky-like deviation of the heat capacities between the compounds themselves is discussed elsewhere. ${ }^{(6)}$ 


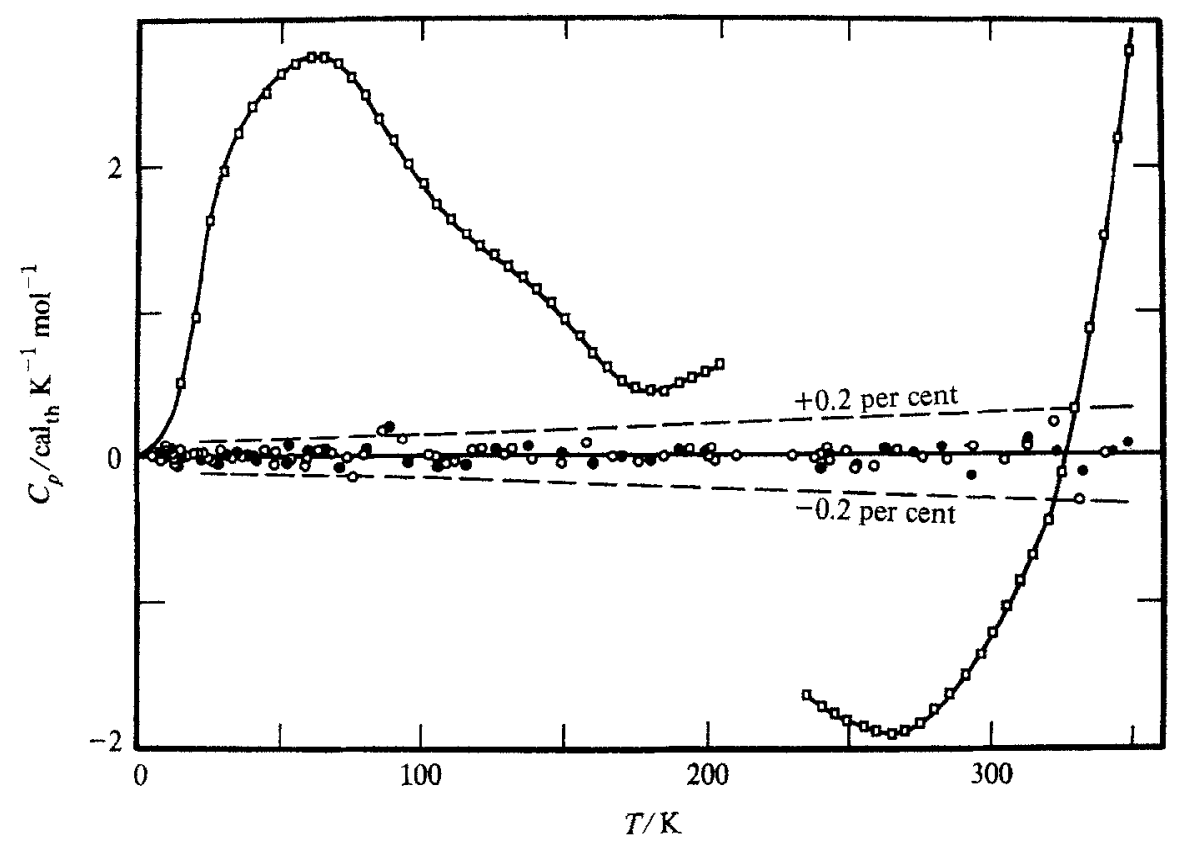

FIGURE 3. Deviation plot for BEAN and BEAZ.

$\square, C_{p}$ (BEAN, smoothed) - $C_{p}$ (BEAZ, smoothed); $O, C_{p}$ (BEAN, exptl)- $C_{p}$ (BEAN, smoothed); -, $C_{p}$ (BEAZ, exptl) - $C_{p}$ (BEAZ, smoothed).

The dashed lines indicate deviations of \pm 0.2 from the smoothed curves.

\section{BIFURCATION OF THE TRANSITIONS}

Although only one series of quasi-equilibrium points has been taken through the transition region of BEAN, the existence of the bifurcation is indicated in other ways. A series of rapidly collected points (which involved heating for $10 \mathrm{~min}$, waiting $60 \mathrm{~min}$, and repeating) also showed a drop in the heat capacity at $222.3 \mathrm{~K}$. In addition, the rate of equilibration is distinctly different on the two peaks, and is much slower on the low-temperature peak. Finally, the integrated enthalpy from the $C_{p}$ curve agrees with the value of $\Delta H_{t}$ provided the minimum produced by bifurcation is included in the curve.

The long equilibration times in the transition regions of both compounds require discussion. Following an energy input (usually sufficiently large to raise the sample temperature by several $\mathrm{K}$ in the non-transition region) the sample would require more than several days to reach internal thermal equilibrium. In contrast, outside the transitions, equilibrium was usually reached within 20 min following energy inputs which were five times as intensive.

Such slow equilibrium times and hysteresis have previously been reported ${ }^{(10,11)}$ for other ammonium salts. Sorai and coworkers ${ }^{(11)}$ as well as Alles et al. ${ }^{(12)}$ found the equilibrium times for $\mathrm{NH}_{4} \mathrm{Br}$ increased from $10 \mathrm{~min}$ to several hours near the first-order phase transition near $108 \mathrm{~K}$. 
Due to the long equilibrium times no enthalpy measurements could be made between the peaks. When energy sufficient to raise the temperature from an initial value well below the transitions to in between the peaks was added, the sample nearest the heater would heat through both transitions and then undercool as it came into equilibrium with the rest of the sample. The resulting temperature would be lower than that of the lower peak. Further addition of energy would reveal a heat capacity very nearly that of the lattice. Enthalpy measurements of the transitions were thus made only across the entire transition regions for both compounds.

The very high values of the transitional heat capacities are indicative of a firstorder transition. This first-order nature is confirmed by other evidence: hysteresis effects have been observed near the transitions, and substantial volumetric changes occurred within the samples during the measurements. One example of hysteresis has been mentioned above. Another occurred when the nickel compound was cooled slowly from above the transitions to $207 \mathrm{~K}, 15 \mathrm{~K}$ below the transitions. Subsequent heating of the sample revealed less than 10 per cent of the transitional enthalpy indicating the sample had undercooled.

For both compounds, the small crystals which had been loosely loaded into the calorimeter were found to be tightly packed fine powders during unloading. Such shattering must have taken place at the transitions and is characteristic of first-order transitions.

\section{COMPARISON WITH OTHER COMPOUNDS}

The thermal properties of the series of compounds $\left[\left(\mathrm{CH}_{3} \mathrm{CH}_{2}\right)_{4} \mathrm{~N}\right]_{2} \mathrm{MCl}_{4}$, where $\mathrm{M}=\mathrm{Mn}, \mathrm{Fe}, \mathrm{Ni}, \mathrm{Co}, \mathrm{Cu}$, and $\mathrm{Zn}$, have been previously studied by Melia and Merrifield $^{(13)}$ who used differential scanning calorimetry (d.s.c.). They found two distinct transitions in the manganese compound and shoulders on the main peaks of the cobalt and zinc compounds. The nickel, iron, and copper compounds revealed only a single anomaly. A comparison of their results with the present work is shown in table 5. It is found that the transition in BEAN observed with d.s.c. was several $\mathrm{K}$

TABLE 5. Temperatures, enthalpies, and entropies of transition ${ }^{a}$ $\left(\mathrm{cal}_{\mathrm{th}}=4.184 \mathrm{~J}\right)$

\begin{tabular}{|c|c|c|c|c|}
\hline Compound $^{b}$ & $\frac{T_{\mathrm{t}}}{\mathrm{K}}$ & $\frac{\Delta H_{\mathrm{t}}}{\mathrm{cal}_{\mathrm{th}} \mathrm{mol}^{-1}}$ & $\frac{\Delta S_{\mathrm{t}}}{\mathrm{cal}_{\mathrm{th}} \mathrm{K}^{-1} \mathrm{~mol}^{-1}}$ & References \\
\hline $\mathrm{R}_{2} \mathrm{NiCl}_{\mathbf{4}}$ & $\begin{array}{l}222.1 \\
222.7 \\
{[218]}\end{array}$ & $\begin{array}{c}2087 \\
{[1760]}\end{array}$ & $\begin{array}{c}9.16 \\
{[8.1]}\end{array}$ & $\begin{array}{c}\text { This work } \\
\text { (13) }\end{array}$ \\
\hline $\begin{array}{r}\mathrm{R}_{2} \mathrm{ZnCl}_{\mathbf{4}} \\
\text { (heating) }\end{array}$ & $\begin{array}{l}227.9 \\
228.9\end{array}$ & 2284 & 9.96 & This work \\
\hline $\begin{array}{l}\text { (heating) } \\
\mathrm{R}_{2} \mathrm{MnCl}_{4}\end{array}$ & $\begin{array}{c}{[227]} \\
218 \\
224\end{array}$ & $\begin{array}{c}{[2120]} \\
1120 \\
780\end{array}$ & $\begin{array}{l}{[9.3]} \\
5.1 \\
3.5\end{array}$ & $\begin{array}{l}\text { (13) } \\
(13)\end{array}$ \\
\hline & & 1900 & 8.6 & \\
\hline
\end{tabular}

${ }^{a}$ Results in [] are d.s.c. determinations.

${ }^{\circ} \mathrm{R}=\left(\mathrm{CH}_{3} \mathrm{CH}_{2}\right)_{4} \mathrm{~N}$. 
lower in temperature than the present work and that the entropy of transition was lower; presumably the long equilibration time and rapid heating rate $(4 \mathrm{~K} / \mathrm{min})$ were the causes of these discrepancies. Had the sample been cooled through the transitions too rapidly, some of the material would have undercooled, remaining in the hightemperature phase. When the sample was then heated, not all of the normal transitional enthalpy would be required; hence, a low value for the entropy would be found.

The two studies are in closer agreement for the zinc compound. This is expected since both the equilibration times for the BEAZ are shorter than those of BEAN and the two-phase transitions occur $1 \mathrm{~K}$ apart, rather than only $0.6 \mathrm{~K}$ apart in BEAN. Consequently, the d.s.c. was able to detect two peaks (at about $213 \mathrm{~K}$ and $218 \mathrm{~K}$ ) during cooling. This separation may mean that one of the processes occurring within BEAZ is more susceptible to undercooling. During heating a single peak was observed at $227 \mathrm{~K}$.

\section{MECHANISM OF THE TRANSITION}

The $300 \mathrm{~K}$ X-ray diffractional determination of BEAN $^{(2)}$ structure revealed several types of disorder. The methylene carbon atoms are rotated approximately $\pi / 4$ from the two mirror planes which intersect at the nitrogen position. As the methylene carbons flop from side to side the tetraethylammonium groups form motionally disordered right- and left-handed swastikas. Some disorder is also revealed in the $\mathrm{NiCl}_{4}^{2-}$ groups by the abnormal thermal quantities.

The temperature dependence of the magnetic anisotropies of BEAN has been measured by Gerloch and Slade. ${ }^{(3)}$ The anisotropies reveal a large discontinuous change near $220 \mathrm{~K}$. Thermal hysteresis was observed during these measurements. As the authors note, it has been previously observed ${ }^{(14)}$ that slight structural changes can cause large alterations of the magnetic anisotropies. Such structural changes were indicated by Gerloch and Slade's analysis of the prior magnetic susceptibilities of BEAN collected by Figgis and coworkers. ${ }^{(15)}$ The data in the two regions above and below the transition were fitted to a three-parameter model, involving $\theta$ (the $\mathrm{Cl}-\mathrm{Ni}-\mathrm{Cl}$ angle), $\lambda$ (the spin-orbit coupling parameter), and $k$ (the electron-delocalization parameter). The low-temperature angle was fitted as $109 \pi / 180$, (i.e. almost perfectly tetrahedral) while above the transition $\theta$ was taken as $106 \pi / 180$, in good agreement with the crystallographic value. ${ }^{(2)}$ The high-temperature value of $\lambda$ was taken as $190 \mathrm{~cm}^{-1}, 25$ per cent larger than the low-temperature value.

The specific causes of the twin transitions $\left[\left(\mathrm{CH}_{3} \mathrm{CH}_{2}\right)_{4} \mathrm{~N}\right]_{2} \mathrm{MCl}_{4}$ are not presently understood. The several types of motional disorder as well as the volumetric changes are certainly all significant but the amount of entropy each process contributes cannot yet be determined. Temperature-dependent structural studies are obviously desirable. Since Melia and Merrifield ${ }^{(13)}$ have found the peaks in the heat capacity of the manganese compound to be separated by $6 \mathrm{~K}$, this substance would be the logical candidate with which to begin.

Some suggestions can be made on the basis of the present results. First-order transitions occur during reorientation of symmetric groups such as the ammonium group in $\mathrm{NH}_{4} \mathrm{Br},{ }^{(11,12)}$ and in $\mathrm{NH}_{4} \mathrm{ZnF}_{3}$ and $\mathrm{NH}_{4} \mathrm{CoF}_{3}{ }^{(10)}$ In the latter pair of compounds, reorientation accompanies a structural change, which expands the 
lattice sufficiently to allow the reorientation. Since the tetraethylammonium groups would presumably require more space for their disorder, we assume this is the disorder which sets in at the structural change. The consequent motion of the ammonium groups may then allow the $\mathrm{MCl}_{4}^{2-}$ groups to disorder. The long equilibration times may be a consequence of the two types of tetrahedral groups interfering with one another's motion. An adiabatic thermophysical study of the manganese compound and its equilibrium time behavior may yield important information.

Several other people have also contributed to the present work. We wish to acknowledge Dr James Thirtle for the preparation and analyses of the samples and $\mathrm{Mr}$ Charles Galeas for his assistance with the collection of results for BEAZ and both him and Ms Laurel Harmon for the computer treatment and reduction of the results. We also wish to thank Professor T. M. Dunn for helpful discussion.

\section{REFERENCES}

1. Ham, F. S. Phys. Rev. 1965, 138, B1727.

2. Stucky, G. D.; Folkers, J. B.; Kistenmacher, T. J. Acta Cryst. 1967, 23, 1064.

3. Gerloch, M.; Slade, R. C. J. Chem. Soc. 1969, 91, 1022.

4. Clark, K. J. H.; Dunn, T. M. J. Chem. Soc, 1963, 85, 1198.

5. Hanson, J. Personal Communication cited by Koester, V. J.; Dunn, T. M. Inorg. Chem. 1975, $14,1811$.

6. Landee, C. P.; Westrum, E. F. Jr.; Dunn, T. M. To be published.

7. Gill, N. S.; Nyholm, R. S. J. Chem. Soc. 1959, 81, 3997.

8. Westrum, E. F. Jr.; Furukawa, G. T.; McCullough, J. P. Adiabatic Low-temperature Calorimetry. In Experimental Thermodynamics Vol. 1. McCullough, J. P.; Scott, D. W.: editors. Butterworths: London. 1968.

9. Stimson, H. F. J. Res. Nat. Bur. Stds. (U.S.) 1961, 65, A784. In Temperature, Its Measurement and Control in Science and Industry. Herzfeld, C.: editor. Reinhold: New York. 1962. p. 59.

10. Bartolome, J.; Navarro, R.; Gonzalez, D.; de Jongh, L. J. J. Physica 1977, 92, B23.

11. Sorai, M.; Suga, H.; Seki, S. Bull. Chem. Soc. Japan 1965, 38, 1125.

12. Alles, A.; Bartel, J. J.; Chavret, M.; Westrum, E. F., Jr. Proceedings of fourth international conference on chemical thermodynamics 1975, II, 58.

13. Melia, J. P.; Merrifield, R. J. Chem. Soc. 1976, 98, A1166.

14. Bently, R. B.; Gerloch, M.; Lewis, J.; Quested, P. N. J. Chem. Soc. 1971, 23 , A3751.

15. Figgis, B. N.; Lewis, J.; Mabbs, F. E.; Webb, G. A. J. Chem. Soc. 1966, 10, A1411.

$\left(\mathrm{Et}_{4} \mathrm{~N}\right)_{2} \mathrm{NiCl}_{4}, 5964-71-6$

Registry Nos.

$\left(\mathrm{Et}_{4} \mathrm{~N}\right)_{2} \mathrm{ZnCl}_{4}, 5964-74-9$

\section{Appendix}

The sluggish nature of the transitions in the two compounds made true equilibrium measurements difficult. The results detailing the shapes of the transitional heatcapacity peaks were obtained without waiting for ultimate equilibrium. Rather, as is explained below, use was made of the regularity of equilibration behavior to estimate the final equilibrium temperatures on most of the determinations.

Preliminary studies of the transitions showed that following an energy input, the rate of change of temperature was almost linear with periods greater than $1 \mathrm{~d}$. Such linearity was observed for both compounds even though, for times less than $1 \mathrm{~d}$, the equilibration rates were different for each of the peaks. The transition results were 
collected by establishing zero drift in regions adjacent to the transition where hysteresis did not occur, then making an energy input, and observing the rate of change of the temperature of the sample until this rate followed the "normal" curve. Since the "apparent drift" was then largely occasioned by relaxation processes, it is possible to predict the equilibrium temperature; no more observations were necessary on most of the determinations and the next energy input was initiated.

The refinement of results for each determination consisted of making a tentative estimate of the slight deviations from adiabaticity on the basis of an assumed heat capacity, then deducing the estimated equilibration time, and finally extrapolating the equilibration rate to calculate the additional temperature increment had the drift been carried to equilibrium. This increment was subtracted from the last temperature measured. Then the increments between fore- and after-drift temperatures were used to deduce an apparent heat capacity. A revised adjustment was made for deviations from adiabaticity, new final equilibrium times were estimated, and revised heat capacities deduced. Convergent values for the heat capacities were always produced by three iterations; most converged after two. The heat capacities were considered to have converged when further iteration changed the heat capacity by less than 5 per cent. The estimate of the additional temperature change required for equilibrium accounted for 80 per cent of the total correction with the other 20 per cent due to adjustments for nonadiabaticity.

The ultimate accuracy of these measurements depends on the validity of the assumption of a consistent equilibration behavior for all points as the basis for the extrapolation routine. One test of this assumption is the comparison between the integrated area under the curve specified by these points and the enthalpy derived from the enthalpy determinations. The agreement is within 2.5 per cent. Still, points on the transition curve must be considered to have an uncertainty of twice this value. 